Original article

\title{
Use of the GLYCEMIZER ${ }^{\circledR}$ tool by general practitioners to meet individual glycated hemoglobin goals in patients with type 2 diabetes mellitus
}

\author{
Nicolas Byron Hatziisaak, Telemachos Hatziisaak, Urs Keller \\ PizolCare Medical Network, St. Gallen, Switzerland
}

Received 15 July 2020, Revised 6 February 2021, Accepted 20 April 2021

C 2020, Hatziisaak N.B., Hatziisaak T., Keller U.

(C) 2020, Russian Open Medical Journal

\begin{abstract}
Background - For general practitioners (GPs), it is often not easy to determine the individual glycated hemoglobin (HbA1c)-goal of patients with type 2 diabetes mellitus (T2DM) in order to offer them a tailored treatment and minimize side effects. Usually, they simply rely on their gut feeling.

Objective - We assessed the usefulness of an easy-to-use algorithm (GLYCEMIZER ${ }^{\circledast}$ ) to calculate individual HbA1c-goals and compared them with targeted ('gut feeling' of the GP's) and achieved levels.

Material and Methods - In this cross-sectional survey, general practitioners were asked to report anonymized data of at least 30 consecutive patients with T2DM presenting in their offices from May $1^{\text {st }}$ to August $15^{\text {th }} 2016$ after obtaining informed consent. Demographic, clinical and biochemical data were used for the GLYCEMIZER ${ }^{\circledR}$ tool to calculate the individual HbA1c-goals. A statistical analysis was conducted in order to compare the calculated HbA1c-goals with targeted and achieved HbA1c-levels.

Results - A total of 184 patients (mean age: 69y) were enrolled by 6 participating general practitioners from the Werdenberg-Sarganserland region in eastern Switzerland. Four patients did not meet the inclusion criteria. The overall median calculated HbA1c-goal did not differ from the targeted and achieved levels ( $7.1 \%$ vs. $7.0 \%$ vs. $7.1 \%, p=0.894)$. There was a significant difference between achieved and calculated $\mathrm{HbA1c}$ levels in patients aged $<50$ ( $n=13$, median $7.2 \%$ vs. $6.5 \%, p=0.014)$, goals not achieved) and patients aged $>71$ ( $n=85$, median $6.9 \%$ vs. $7.5 \%$, $\mathrm{p}=0.005$ ), lower levels achieved in relation to calculated HbA1c-goals). Both in patients treated with insulin ( $n=44)$ and in patients without insulin ( $n=136)$ the achieved HbA1c-levels met the calculated goals (no insulin: $6.9 \%$ vs. $7.0 \%$, ns; with insulin: $7.8 \%$ vs. $7.7 \%$, ns). In regard to CKD-stages 3 and 4 the achieved HbA1c-levels were significantly lower than calculated ( $n=41$, median $6.9 \%$ vs. $7.6 \%, p=0.001$ ).

Conclusion - Calculating HbA1c-goals using the GLYCEMIZER tool is more accurate than relying on gut feeling alone, and is specifically useful in the treatment of patients with T2DM of less than 50, as well as more than 70 years of age. Furthermore, it is helpful to meet individual HbA1c-goals in patients with CKD-stages 3+.
\end{abstract}

Keywords: type 2 diabetes mellitus, glycated hemoglobin, GLYCEMIZER, general practice.

Cite as Hatziisaak NB, Hatziisaak T, Keller U. Use of the GLYCEMIZER ${ }^{\circledR}$ tool by general practitioners to meet individual glycated hemoglobin goals in patients with type 2 diabetes mellitus. Russian Open Medical Journal 2021; 10: e0207.

Correspondence to Telemachos Hatziisaak. Phone: +41 8178319 72. E-Mail: tele.hatziisaak@bluewin.ch

\section{Background}

Since the introduction of HbA1c (glycated hemoglobin) as a parameter for the characterization of diabetes control, the perception has shifted from a rigid interpretation to an individual one. After the UKPDS trial the general practitioner's goal was to achieve an HbA1c equal or below 7\% for his patients with T2DM (type 2 diabetes mellitus), keeping in mind that microvascular complications of diabetes were efficiently reduced only with levels below $6.5 \%$ [1]. However, the ACCORD study showed that "the lower the better" was not suitable in the treatment of T2DM, as lower $\mathrm{HbA1C}$ levels were correlated to higher mortality [2]. And although intensive blood glucose lowering showed a beneficial effect on avoiding end stage kidney disease in the long run [3], lowering mortality was rather achieved by disease awareness and active cardiovascular risk factor control adapting a healthy lifestyle [4]. In 2013, Inzucchi et al. published a new approach to obtain optimal HbA1c-goals in patients with T2DM. These individualized goals have found a broad acceptance within both diabetologists and general practitioners in Switzerland and were therefore introduced into national guidelines. 2015 an update of the new treatment guidelines was published [5]. Using these recommendations Merck Sharp and Dome (MSD Switzerland) together with Roger Lehmann from the endocrinology and diabetology department of the University Hospital of Zurich created an algorithm to calculate the optimal HbA1c-levels in order to support GPs (general practitioners) in daily decision making. This tool, a decent computer program, named GLYCEMIZER ${ }^{\circledR}$ was first presented to the GPs in 2015.

As T2DM is a frequent disease seen in general practice, our group of GPs wanted to assess the usefulness and feasibility of the GLYCEMIZER $^{\circledast}$ tool to calculate individualized HbA1c-goals in daily work. We also aimed at comparing the calculated goals to the achieved HbA1c-levels in a population of patients with T2DM at different stages of their disease. Furthermore, we wanted to 
examine, if the calculated goals and achieved levels were correlated to targeted goals, i.e., the practitioner's experience (gut feeling).

\section{Material and Methods}

11 general practitioners from the PizolCare medical network were randomly chosen and asked to participate in this study. The PizolCare medical network is a managed care institution with budget responsibility, located in rural eastern Switzerland. It is a small network, which comprises 106 doctors of whom 44 are GPs.

The GPs were asked to fill in a short questionnaire form (see Appendix) after every consultation with a patient with T2DM and fax it to the study office. The data needed to feed the GLYCEMIZER were gender, age, diabetes duration and serum creatinine. Furthermore, they had to tick yes-or-no-boxes for (diabetes associated) comorbidities, use of insulin or sulfonylureas, glinides respectively, and professional nursing assistance. The GPs then had to report the past four HbA1c-levels obtained and they had to spontaneously determine a specific individual HbA1c-target for each patient.

The data were collected completely anonymously with the GPS adding a consecutive number to the fax form. GPs were provided with a logbook, were they kept the corresponding patient data for their own. In case of lacking information, they were able to quickly provide them.

Data collection took place from May, $1^{\text {st }}$ to August, $15^{\text {th }} 2016$. The data provided were transferred to an excel-file. eGFR (estimated Glomerular Filtration Rate) was calculated for each patient using the CKD-EPI formula (Chronic Kidney Disease). The GLYCEMIZER calculator was fed with the necessary data and calculated $\mathrm{HbA1C}$ goals were retrieved. Three columns were assigned: targeted (gut feeling), achieved (using the mean value of the four reported $\mathrm{HbA1c}$-levels), and calculated $\mathrm{HbA1c}$.

We then conducted a comparison between these three values under different angles. An overall analysis was made. At the disclosure of the doctors taking part in this study, an individual analysis for every doctor was made. Further on, we conducted several subgroup analyses in relation to age, diabetes duration, insulin use and renal function. For every topic, we calculated the median and the $25^{\text {th }}$ and $75^{\text {th }}$ percentile, thus creating three comparing boxplot graphs for better visualization, using Microsoft Excel 2010. Mean values, standard deviation and confidence intervals were calculated. Doublesided Student's t-tests were performed in order to detect significant differences between the three HbA1c-levels.

\section{Results}

A total of 184 CRFs (Case Report Files) were sent to the study office by 6 contributing GP's. 4 CRFs were excluded from the analysis. 2 CRFs referred to individuals who obtained their diagnosis of T2DM within less than one year, thus making it impossible to determine a reliable achieved $\mathrm{HbA1c}$, because of a lack of homeostasis. One CRF indicated that the patient was not tested for $\mathrm{HbA} 1 \mathrm{c}$ for two years. Finally, one CRF suggested that the patient suffered rather from LADA then T2DM. The baseline characteristics are shown in Table 1.

The mean value of the overall HbA1c target was $7.0 \%(p=0.984$, Cl $95 \%=7.1 \%-7.3 \%$ ). The achieved levels matched exactly the calculated ones with a median of $7.1 \%$, while the range between the $25^{\text {th }}$ and the $75^{\text {th }}$ percentile was wider in the 'achieved' group than in the 'calculated' group, as can be seen on Figure 1. There was no significant difference between female $(n=74)$ and male $(n=106)$ patients regarding $\mathrm{HbA} 1 \mathrm{c}$-levels when analyzed in their specific subgroup (Figure 2). The trend to slightly higher HbA1c-levels in women compared to men was owed to higher age, longer duration of diabetes and higher CKD-stages (Chronic Kidney Disease). In patients aged less than 50 years (Figure $3, n=13$ ) we found a significant difference between the achieved and calculated HbA1clevels $(7.3 \%$ vs. $6.5 \%, \mathrm{p}=0.014, \mathrm{Cl} 95 \%=6.8 \%-7.9 \%$, vs. $6.4 \%-$ $6.7 \%)$. Patient in this group achieved higher levels than calculated with the GLYCEMIZER. In contrast to these results in patients aged more than 71 years (Figure 4, n=85) the achieved levels were significantly lower than the calculated ones (mean values $7.2 \%$ vs. $7.5 \%, p=0.005, \mathrm{Cl} 95 \%=7.0 \%-7.4 \%$ vs. $7.4 \%-7.6 \%$ ). No significant difference between achieved and calculated $\mathrm{HbA1clevels}$ was observed in patients aged 51 to 70 (7.2\% vs. $7.0 \%, p=0.082$ ).

Patients with T2DM and normal or stage 1 or 2 renal dysfunction ( $n=139$ ) according to the CKD-classification (i.e., eGFR $>60 \mathrm{ml} / \mathrm{min} / 1.73 \mathrm{~m} 2$ ) showed no difference between the achieved HbA1c-levels and the calculated values $(7.2 \%$ vs. $7.1 \%, p=0.07)$, whereas patients with CKD-stages 3 and 4 (eGFR 15 $60 \mathrm{ml} / \mathrm{min} / 1.73 \mathrm{~m} 2$ ) showed significantly lower HbA1c-levels than calculated with GLYCEMIZER (Figure $5, \mathrm{n}=41$, mean values $7.1 \%$ vs. $7.6 \%, p=0.001, \mathrm{Cl} 95 \%=6.8 \%-7.4 \%$ vs. $7.5 \%-7.7 \%$ ).

We did not find any significant difference between achieved and calculated HbA1c-levels in patients treated with or without insulin, when analyzed within the specific groups (Figure 6). When comparing the two groups, we detected a significant difference between the HbA1c-levels achieved in the insulin- and no-insulin-group (mean values $7.8 \%$ vs. $7.0 \%, p=0.03$ ). Patients receiving insulin had significantly higher HbA1c-levels compared to patients without insulin treatment. There was no significant difference between the calculated HbA1c-levels (mean values $7.6 \%$ vs. $7.1 \%, p=0.078$ ). Further on, we did not detect a difference between achieved and calculated HbA1c-levels in relation to diabetes duration in the three different subgroups (diabetes duration $<5 y, 6-15 y$, $>15 y$ ). Patients with a diabetes duration of less than 5 years achieved a mean $\mathrm{HbA1c}$ of $6.8 \%$ vs. $6.9 \%$ when calculated with GLYCEMIZER ( $p=0.483$ ). Patients with a history of T2DM lasting between 6 and 15 years achieved a mean $\mathrm{HbA} 1 \mathrm{c}$ of $7.3 \%$ (HbA1c calculated with GLYCEMIZER: 7.1\%; $p=0.222$ ). Finally, patients with a diabetes duration of more than 15 years showed a mean $\mathrm{HbA} 1 \mathrm{c}$ of $7.5 \%$ vs. $7.6 \%$ when calculated with GLYCEMIZER $(p=0.303)$.

Table 1. Baseline characteristics

\begin{tabular}{lcccccc}
\hline & Male & $(\%)$ & Female & $(\%)$ & Total $\varnothing$ & $(\%)$ \\
\hline Patients & 106 & 58.9 & 74 & 41.1 & 180 & 100 \\
Age $\varnothing \mathrm{y}$ & 66 & & 72 & & 69 & \\
Age $<50 \mathrm{y}$ & 9 & 8.5 & 4 & 5.4 & 13 & 7.2 \\
Age 51-70y & 57 & 53.8 & 25 & 33.8 & 82 & 45.6 \\
Age $>71 \mathrm{y}$ & 40 & 37.7 & 45 & 60.8 & 85 & 47.2 \\
T2D duration $\varnothing \mathrm{y}$ & 9.5 & & 11 & & 10 & \\
T2D duration $<5 \mathrm{y}$ & 38 & 35.9 & 22 & 29.7 & 60 & 33.3 \\
T2D duration 6-15y & 47 & 44.3 & 29 & 39.2 & 76 & 42.2 \\
T2D duration $>16 \mathrm{y}$ & 21 & 19.8 & 23 & 31.1 & 44 & 24.4 \\
Insulin use & 25 & 23.6 & 19 & 25.7 & 44 & 24.4 \\
CKD 1, 2 & 93 & 87.7 & 46 & 62.2 & 139 & 77.2 \\
CKD 3, 4 & 13 & 12.3 & 28 & 37.8 & 41 & 22.8 \\
\hline
\end{tabular}

Women included in this cross-sectional study were older, had a longer history of diabetes and more frequently an impaired renal function. Standard deviation for age was 10.3 years for women, 9.9 years for men and 10.5 years overall. Standard deviation for diabetes duration was 7.6 years for women, 6.8 years for men and 7.2 years overall. No significant differences were detected in all compared data regarding gender and disease characteristics. 


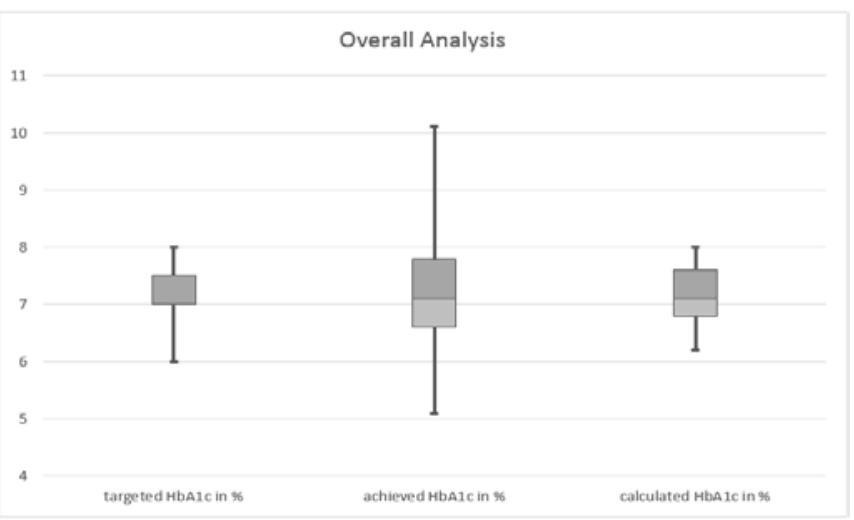

Figure 1. Overall analysis $(n=180)$. This boxplot displays the distribution of the HbA1c levels comprising all patients in this study. The median of the three examined $\mathrm{HbA1c}$-values (targeted, achieved and calculated) reaches $7.0 \%$ vs. $7.1 \%$ vs. $7.1 \%$. The box represents the mean $50 \%$ of the data. The lower limit is the $25^{\text {th }}$ percentile, the upper one the $75^{\text {th }}$ percentile. The median is the bar in the middle. The whiskers show the minimal and maximal value respectively.

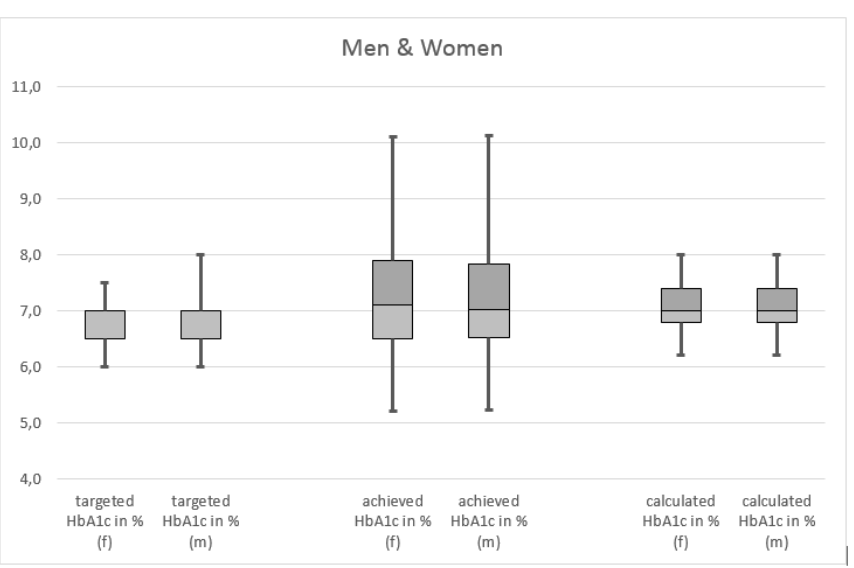

Figure 2. Comparison between female and male patients. No significant difference between men and women are observed regarding achieved HbA1c-levels.

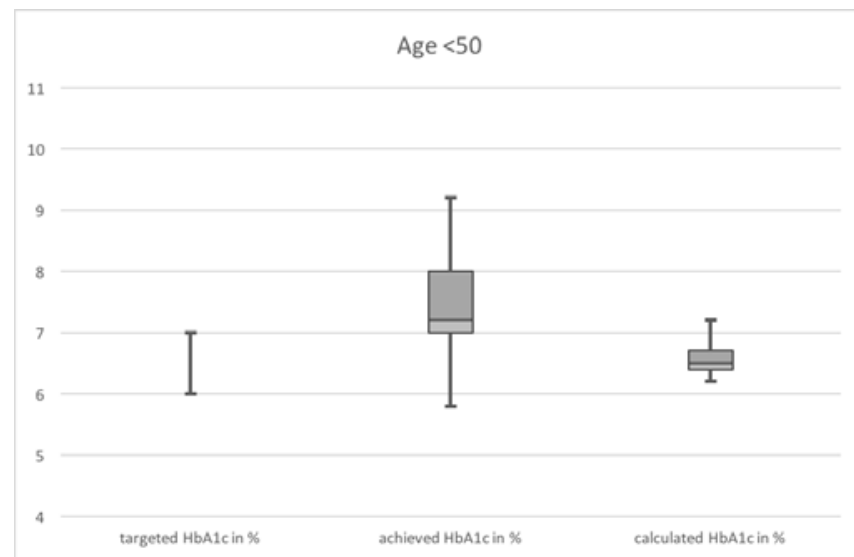

Figure 3. Patients $<50$ years old $(\mathbf{n}=\mathbf{1 3})$. The achieved HbA1c-levels show a median of $7.3 \%$, whereas the calculated values amount only to a median of $6.5 \%$

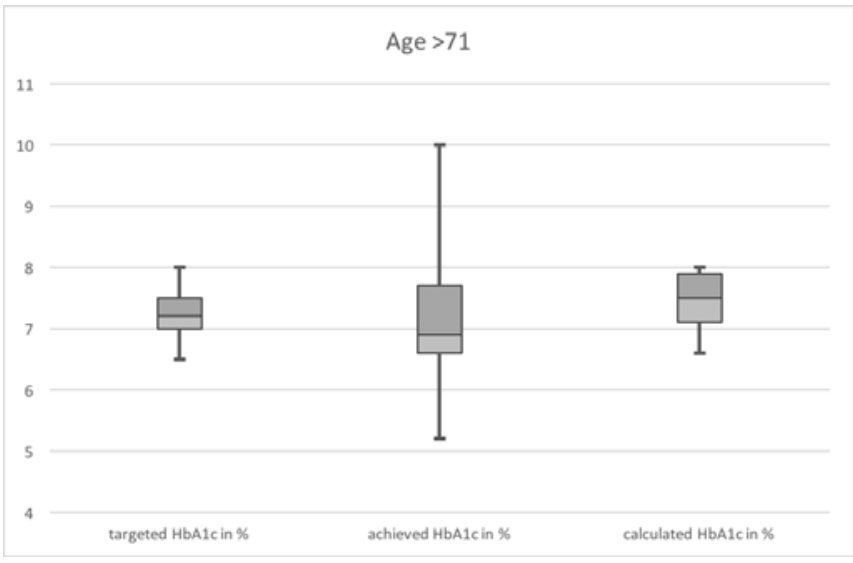

Figure 4. Patients $>\mathbf{7 1}$ years old $(\mathbf{n}=\mathbf{8 5})$. Note that the median of achieved HbA1c is $6.9 \%$, whereas the calculated one using GLYCEMIZER comes to $7.5 \%$

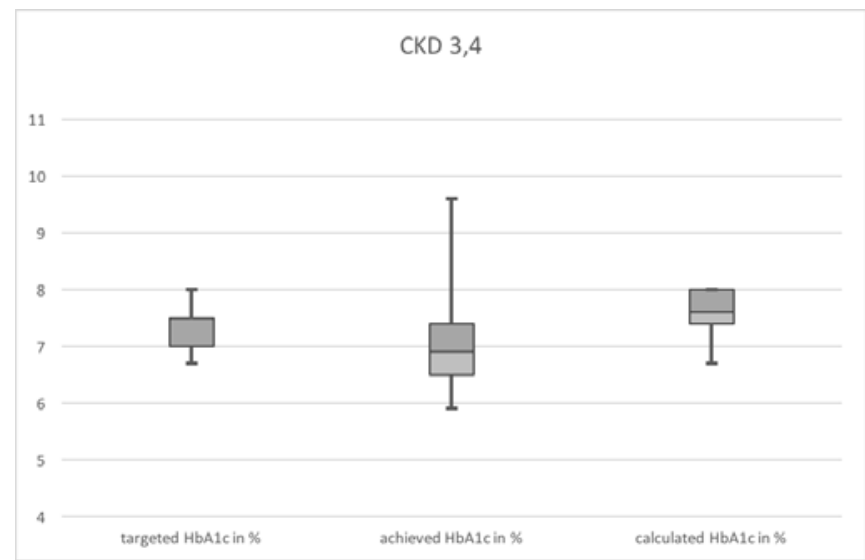

Figure 5. Patients with renal dysfunction CKD 3,4 ( $n=41)$. The median HbA1c reaches $6.9 \%$. Calculated with GLYCEMIZER, it should amount to $7.6 \%$.

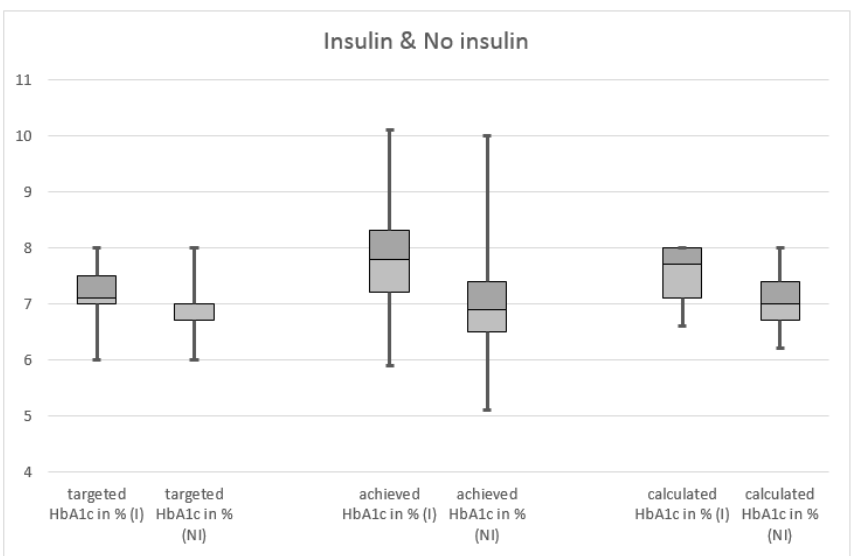

Figure 6. Patients treated with insulin $(n=44)$ vs. no insulin $(n=136)$. No significant differences were observed between achieved and calculated HbA1c-levels within the specific groups. On the other hand, there is a significant difference between the achieved HbA1c-levels when the insulin group is compared with the non-insulin group. 


\section{Discussion}

In this study, we wanted to determine the usefulness and feasibility of the GLYCEMIZER calculator to achieve individual $\mathrm{HbA1c}$-goals, as suggested by literature, in patients with T2DM in general practice. Furthermore, we wanted to examine if there was a difference between the targeted, achieved and calculated individualized $\mathrm{HbA} 1 \mathrm{c}$ levels.

As evidence has emerged that GPs in Switzerland do not sufficiently comply with guidelines in order to achieve better outcomes for patients with T2DM, thus causing significantly more hospitalizations [6], we wanted to shed a light on a part of these findings. In a rural region of eastern Switzerland, situated in the Canton of St. Gallen, our medical network provides medical services to approximately $60^{\prime} 000$ inhabitants. With a prevalence of $4.9 \%$ for diabetes in Switzerland our network cares for an estimated 3000 patients suffering mainly from T2DM. In this study 6 GP's enrolled 180 patients with T2DM attempting to determine, whether GPs achieved the targeted HbA1c-levels and whether the achieved levels were matching the calculated individual levels using the GLYCEMIZER tool.

The analysis of the complete data set showed no significant difference between achieved and calculated HbA1c-levels. This was the case regardless of gender, age, duration of diabetes, use of insulin and renal dysfunction. These findings come in accordance to a previous analysis in Switzerland, which showed similar results in terms of mean achieved HbA1c-levels and use of insulin [7]. In the last years, many observational studies to evaluate glycemic control have been conducted in different countries. A study from Australia focusing on patients with type-2diabetes in general practice showed that current targets were generally achieved, although a quarter of the patients had clearly poor glycemic control [8]. As this study dates from 2009 one has to take into account that the interpretation of glycemic control was according to the then applicable guidelines, which were rather rigid than dynamic. Nevertheless, the mean achieved $\mathrm{HbA} 1 \mathrm{c}$ in the Greek Panorama study, which was published 2015, was 6.7\%, while the authors noted that one third failed to meet $\mathrm{HbA1C}$ targets [9]. The disease characteristics in this study were comparable to ours. In a Spanish study published $2014 \mathrm{HbA1c}-$ levels were stratified according to disease duration. The results showed a deterioration of glycemic control in the course of 20 years [10]. In our study we confirmed this observation, but we did not find a significant difference between achieved and calculated $\mathrm{HbA1c}$-levels in each analysed subgroup.

Our findings suggest that GPs in our region comply with national guidelines related to the treatment of T2DM when focused on achieving individual HbA1c-goals [5,11]. They seem to be aware of the patient-centered approach in the management of patients with T2DM as described by Inzucchi et al. which is displayed by the GLYCEMIZER tool. This stands in contradiction to the findings of the claims-based cohort study of patients with diabetes, which was published recently. In our study, it was also encouraging to see that GPs are carefully differentiating in the treatment goals of patients treated with insulin or not. The achieved mean HbA1c-level in the group of those treated with insulin was $7.8 \%$, showing no significant difference to the calculated level (7.6\%), but significantly differing from the achieved $\mathrm{HbA} 1 \mathrm{c}$-level in patients not treated with insulin (mean value $7.0 \%, p=0.027$ ). This may be seen as another proof that GPs comply with current guidelines even when it comes to details.
In the subgroup analysis, there were three major findings, which may have an influence on the use of the GLYCEMIZER tool. First, patients with T2DM aged $<50$ years had significantly higher HbA1c-levels than calculated with GLYCEMIZER. This finding is of particular importance, as it is crucial for the diabetic patient to achieve low levels of HbA1c in an early stage of the disease in order to prevent microvascular complications. The result may be biased due to the small number of patients in this group $(n=13)$. Another reason, why the HbA1c-goals are not met in this group may be the relatively short duration of the disease (mean 3.9 years vs. 9.3 years in the group of patients aged $51-70$ and 11.7 years in the group of patients aged $>71$ ). Patients in this group may not have reached a steady state in glycemic control due to various reasons. Nevertheless, we consider this finding very important and think that calculating HbA1c-goals with the GLYCEMIZER tool may be of special usefulness to the GPs in this group of patients aged $<50$ years, who are in an initial stadium of their diabetic career.

Another important observation was the fact that low HbA1clevels were achieved in the group of patients aged $>71$ years $(n=85)$. There was a significant difference between achieved and calculated levels, the mean values being $7.2 \%$ and $7.5 \%$ respectively. This finding comes in accordance to an article, which showed a potential overtreatment of glycaemia in elderly people with T2DM [12]. The authors concluded, that this was owed to the fact, that patients involved in this study were treated according to the rigid guidelines, which were valid at the time the study was conducted (2009-2010). As elderly patients are particularly at risk for hypoglycemic events with potentially dangerous complications it is important for the GP's to keep an eye on the targeted HbA1clevels of this specific group. Using the GLYCEMIZER may be helpful to calculate the optimal HbA1c-goal for patients aged 70 and more.

Finally, we discovered that patients with a compromised renal function in CKD-stages 3 and $4(n=41)$ showed significantly lower HbA1c-levels when compared to the levels calculated with the GLYCEMIZER tool, mean values being $7.1 \%$ and $7.6 \%$ respectively. This finding suggests that patients with T2DM and renal dysfunction are being treated too ambitiously by their GP's. It is well known, that there is a danger for hypoglycemic events in this specific group of patients. Therefore, the use of the GLYCEMIZER tool may be helpful for GPs to avoid possible complications in patients with kidney disease. It requires at least an annual measurement of the serum creatinine level and the calculation of the eGFR (for example using the CKD-EPI formula) to identify patients at risk.

Our study has several strengths and weaknesses. As the sample size is rather small, the results cannot be automatically generalized for the whole diabetic population of Switzerland. Also, there may be a selection bias regarding the contributing GP's. On the other hand, the inclusion time was 3.5 months enabling the participating GPs to enroll a significant part of the diabetics visiting their clinic, thus making the survey representative concerning glycemic control in our particular region.

Calculating the optimal individual HbA1c-goals is a more scientific method than relying on gut feeling and may lead to better results in terms of outcome and quality of life as well as more therapeutic safety in the treatment of patients with T2DM. It takes only one to two minutes to feed the GLYCEMIZER with the data needed, and this can be done during the consultation. 
Our study indicates that despite contrary reports GP's at least in our region of Switzerland seem to be utterly compliant with treatment-guidelines for T2DM. Further studies on a national level are required to prove or dismiss our findings as the current study is limited by its extent.

\section{Conclusion}

We conclude, that using the GLYCEMIZER tool to determine the individual HbA1c-goal for patients with T2DM is helpful for GPs in the following three situations:

1. In patients under 50 years of age,

2. In patients over 70 years of age,

3. In patients with renal dysfunction with impaired eGFR starting from CKD stage 3 and higher.

\section{Ethics approval and consent to participate}

Because of the study being designed as a survey conducted among GPs with the goal to evaluate and compare their skills, while no intervention was performed on patients, approval of the ethics committee was not applicable sensu Swiss law: Bundesgesetz über die Forschung am Menschen (Humanforschungsgesetz, HFG) vom 30. September 2011, Verordnung über die Humanforschung mit Ausnahme der klinischen Versuche (Humanforschungsverordnung, HFV) vom 20. September 2013, Verordnung über klinische Versuche in der Humanforschung (Verordnung über klinische Versuche; KlinV) vom 20. September 2013.

\section{Consent for publication}

All contributing GPs consent for publication.

\section{Conflict of interest}

None declared.

\section{Funding}

This study was done as volunteer work. No funding was necessary.

\section{Acknowledgements}

The authors would like to acknowledge the following fellow general practitioners for contributing to the data collection: Felix Akermann, MD, Buchs SG, Jean-Jacques Brocard, MD, PizolCare-Praxis Sargans, Markus Hitz, MD, Grabs, Claudia Lampert, MD, PizolCare-Praxis Sargans.

\section{Specifications of the PizolCare medical network}

The PizolCare medical network is an integrated medical network, that comprises over 100 doctors (40\% GPs, $60 \%$ specialists) working in private practice environment. The network concludes managed care contracts with various health insurers and shares responsibility for the budget. It is therefore in the interests of the doctors, who work in the network, to provide their patients with the best possible evidence-based medicine in a cost-effective manner. Geographically the PizolCare medical network is situated in the south of the Canton of St. Gallen, Eastern Switzerland. For detailed information please consult the network's website (www.pizolcare.ch)

\section{References}

1. Effect of intensive blood-glucose control with metformin on complications in overweight patients with type 2 diabetes (UKPDS 34). UK Prospective Diabetes Study (UKPDS) Group. Lancet 1998; 352(9131): 854-865. https://pubmed.ncbi.nlm.nih.gov/9742977.

2. ACCORD Study Group, Gerstein HC, Miller ME, Genuth S, Ismail-Beigi F, Buse JB, Goff DC Jr, et al. Long-term effects of intensive glucose lowering on cardiovascular outcomes. N Engl J Med 2011; 364(9): 818828. https://doi.org/10.1056/nejmoa1006524.

3. Wong MG, Perkovic V, Chalmers J, Woodward M, Li Q, Cooper ME, et al. Long-term benefits of intensive glucose control for preventing endstage kidney disease: ADVANCE-ON. Diabetes Care. 2016; 39(5): 694700. https://doi.org/10.2337/dc15-2322.

4. Gaede $P$, Lund-Andersen $H$, Parving $H H$, Pedersen $O$. Effect of a multifactorial intervention on mortality in type 2 diabetes. $N$ Engl J Med 2008; 358(6): 580-591. https://doi.org/10.1056/nejmoa0706245.

5. Inzucchi SE, Bergenstal RM, Buse JB, Diamant M, Ferrannini E, Nauck $M$, et al. Management of hyperglycemia in type 2 diabetes, 2015: a patient-centered approach: update to a position statement of the American Diabetes Association and the European Association for the Study of Diabetes. Diabetes Care 2015; 38(1): 140-149. https://doi.org/10.2337/dc14-2441.

6. Huber CA, Brändle M, Rapold R, Reich O, Rosemann T. A set of four simple performance measures reflecting adherence to guidelines predicts hospitalization: a claims-based cohort study of patients with diabetes. Patient Prefer Adherence 2016; 10: 223-231. https://doi.org/10.2147/ppa.s99895.

7. Gerber PA, Spirk D, Brändle M, Thoenes M, Lehmann R, Keller U. Regional differences of glycemic control in patients with T2DM mellitus in Switzerland: a national cross-sectional survey. Swiss Med Wkly 2011; 141: w13218. https://doi.org/10.4414/smw.2011.13218.

8. Maclsaac RJ, Jerums G, Weekes AJ, Thomas MC: Patterns of glycaemic control in Australian primary care (NEFRON 8). Intern Med J 2009; 39(8): 512-518. https://doi.org/10.1111/j.1445-5994.2008.01821.x.

9. Avramopoulos I, Moulis A, Nikas N. Glycemic control, treatment satisfaction and quality of life in T2DM patients in Greece: The PANORAMA study Greek results. World J Diabetes 2015; 6(1): 208-216. https://doi.org/10.4239/wjd.v6.i1.208.

10. Franch-Nadal J, Roura-Olmeda $P$, Benito-Badorrey B, RodriguezPoncelas A, Coll-de-Tuero G, Mata-Cases M. Metabolic control and cardiovascular risk factors in T2DM patients according to diabetes duration. Fam Pract 2015; 32(1): 27-34. https://doi.org/10.1093/fampra/cmu048.

11. Lehmann R, Bianda T, Brändle M, Czock A., Egli M., Philippe J., et al. Empfehlungen der SGED/SSED: Massnahmen zur Blutzuckerkontrolle bei Patienten mit Diabetes mellitus Typ 2. 2017; 15 p. German. https://www.sgedssed.ch/fileadmin/user upload/6 Diabetologie/61 Empfehlungen_Facharzt/1703_SGED_Empfehlung_BZKontrolle T2DM Finale Version 13 oTabellen.pdf.

12. Müller N, Khunti K, Kuss O, Lindblad U, Nolan JJ, et al. Is there evidence of potential overtreatment of glycaemia in elderly people with T2DM? Data from the GUIDANCE study. Acta Diabetol 2017; 54(2): 209-214. https://doi.org/10.1007/s00592-016-0939-9.

\section{Authors:}

Nicolas Byron Hatziisaak - medical student, PizolCare Praxis Wartau, Truebbach, Switzerland. https://orcid.org/0000-0002-5069-2039.

Telemachos Hatziisaak - MD, Head of PizolCare Praxis Wartau, Truebbach, Switzerland. https://orcid.org/0000-0002-3745-4722.

Urs Keller - MD, Head of PizolCare Praxis Sargans, Sargans, Switzerland https://orcid.org/0000-0003-2833-1525. 


\section{GLYCEMIZER 2016}

Case Report Form

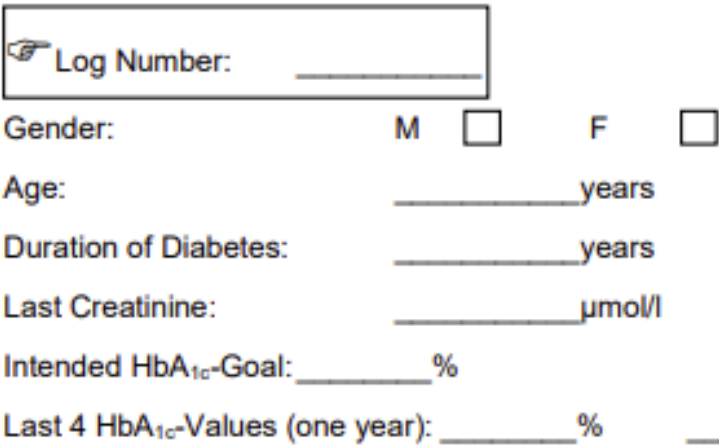
$\%$ $\%$

Please check the applicable boxes:

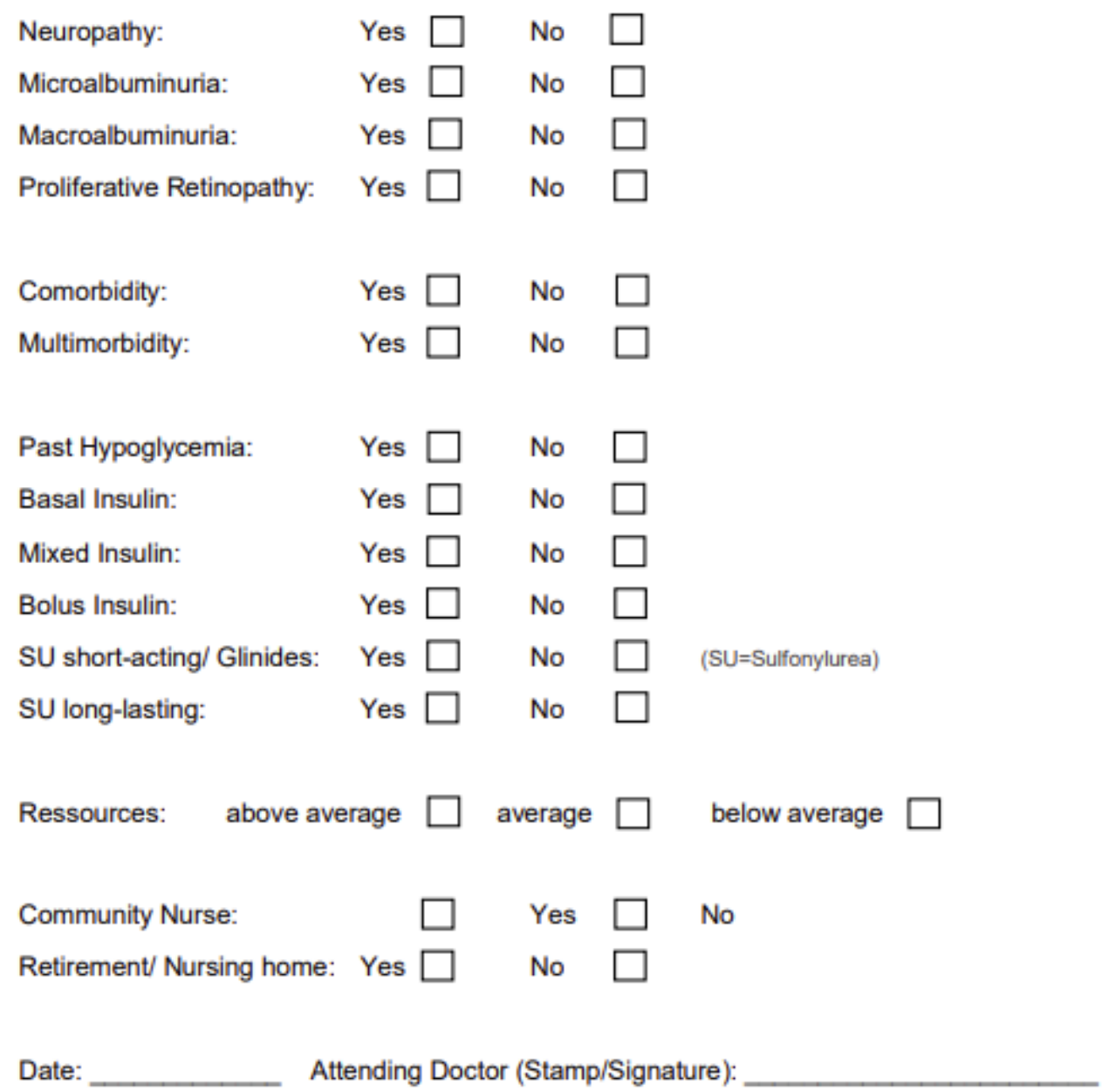

